Research Article

\title{
Study on the Mathematical Model and Propagation Characteristics of AE Waveform Signals during Rock Fracture
}

\author{
Xun You $\mathbb{D}^{1,2}$ Bin Gong $\mathbb{D}^{3},{ }^{3}$ Xin $\mathrm{Lv}\left(\mathbb{D},{ }^{4}\right.$ and Longfei Hu $\mathbb{D}^{2,5}$ \\ ${ }^{1}$ Design \& Research Institute of Masteel Engineering Technology Group Co., Ltd., Maanshan 243000, China \\ ${ }^{2}$ Resources and Environment Engineering Institute, Jiangxi University of Science and Technology, Ganzhou 341000, China \\ ${ }^{3}$ State Key Laboratory of Coastal and Offshore Engineering, Dalian University of Technology, Dalian 116024, China \\ ${ }^{4}$ Modern Technology and Education Center, North China University of Science and Technology, Tangshan 063210, China \\ ${ }_{5}^{5}$ Jiangxi Provincial Society of Engineering Blasting, Nanchang 330031, China \\ Correspondence should be addressed to Bin Gong; gongbin@dlut.edu.cn
}

Received 16 December 2020; Revised 22 January 2021; Accepted 28 January 2021; Published 9 February 2021

Academic Editor: Faming Huang

Copyright (c) 2021 Xun You et al. This is an open access article distributed under the Creative Commons Attribution License, which permits unrestricted use, distribution, and reproduction in any medium, provided the original work is properly cited.

Rock deformation or fracture is accompanied by the phenomenon of acoustic emission (AE). Due to the heterogeneity and anisotropy of rock materials as well as the complexity of their fracture, AE signals recorded by sensors at different positions have different characteristics. To explore factors influencing these differences, this study examines the effects of the physical properties of rocks, such as heterogeneity, anisotropy, and viscosity, on AE waveform signals from the perspective of the rock material and its fracture characteristics as well as the characteristics of the propagation of different AE waveform signals. The results show that the frequency $(f)$ of the AE signals generated by rock fracture is inversely proportional to crack length $(c)$ and directly proportional to the rate of crack growth $(v)$. During signal propagation, the comprehensive effects of such factors as the heterogeneity, anisotropy, and viscosity of rocks as well as environmental noise weaken the energy of the signals and enhance the distribution of signal frequency. Each factor differently influences the time frequency of AE. A model for the propagation of AE signals was built and verified. Finally, as for on-site rock mass engineering, the low-frequency signals should be analysed prior to analysis in rock mass disaster monitoring.

\section{Introduction}

As a typical multiphase composite geological body, rocks are nonuniform, anisotropic, and viscous under the comprehensive action of materials (particles), structure (coupling between particles, diagenetic environment, and transformation), and boundary conditions (stress, temperature, water, and the free boundary) [1-3]. As the fracture of engineering rock mass becomes increasingly complex, the problem of the inaccurate prediction of failure precursors has become increasingly prominent. To simplify models for the analysis of large-scale rock mass failure on-site, the rock mass is regarded as an isotropic body $[4,5]$.

In the 1960s, Lekhnitskii [6] derived the general equation for the anisotropy and elasticity of rock mass from the generalized Hoek law, where this provided the theoretical basis for examining the anisotropy and heterogeneity of rock materials. Methods of inverse analysis using the 3D finite element method (FEM), the Monte Carlo method, Bayesian analytical method, graphic discussion method under the Bragg condition, fractal measurements, and unit step increments in variation have been applied to measure the stress, strain, and displacement of rock media [7-10]. In terms of the anisotropy (including material and stress anisotropy), a certain correspondence has been noted between the velocity ratios of transverse and longitudinal waves and the lithology and geometric distribution of mesoscopic fracture in rocks containing moisture. The presence of moisture enhances the anisotropy of rocks [11-14]. The rate of loading, distribution of coupling, and direction of weak planes further increases the influence of anisotropy and heterogeneity on rock fracture behaviours and should thus 
be considered in examining the engineering stability of rock or rock mass [15]. The separation between particles within rocks and particle fracture can lead to the phenomenon of disproportionation in the propagation of AE signals, such as in the reflection and refraction of the waves, and can even eliminate low-energy signals. Noise enhances the distribution of the frequency signals [16]. The existence of heterogeneity affects their propagation, and anisotropy makes the location of AE signals more difficult $[17,18]$.

The complexity of a rock medium and its rupture can distort the results of monitoring [19]. Research on factors influencing the mechanism of propagation of AE signals can promote the application of AE-based methods to identify rock fracture and can improve the accuracy of prediction of instability-induced fracture in the rock mass.

This study examines the effects of the heterogeneity, anisotropy, and viscosity of rocks as well as noise on their AE waveform. To this end, potential functions for transverse and longitudinal waves under different factors are formulated based on different wave equations in case of AE.

\section{Influence of Rock Material and Its Fracture on Characteristics of AE Propagation}

\subsection{Typical Characteristics of Rock Materials}

2.1.1. Anisotropy. The anisotropy of rocks can be divided into two types [20]. One is caused by the diagenesis of rocks and formed by the directional arrangement of particles and the mutual association among them. It is called material anisotropy. The other is caused by differences in the existence, arrangement, and distribution of microstructural planes, such as pores and microfractures in rocks, which change with the process of fracture. This is called stress anisotropy.

(1) Material Anisotropy. A rock is a typical anisotropic geological body. In the process of diagenesis, macroscopic anisotropy is caused by differences in the arrangement of mineral grains and fracture distribution at the microscopic scale.

(2) Stress Anisotropy. Assuming that the rock is composed of a series of hexahedrons, its stress tensor at any position can be decomposed into a spherical stress tensor with equal stresses in all directions and a deviant stress tensor. The spherical stress tensor causes the volumetric deformation of the rock material and the deviant stress tensor distorts it (deformation and fracture). Stress anisotropy is mainly caused by the deformation, expansion, and interpenetration of pores in rocks. With an increase in stress, the pores undergo a process of "compression $\longrightarrow$ closure $\longrightarrow$ expansion $\longrightarrow$ interconnection." The stress tensor is different in different parts of the rock, which leads to a difference in pore compression or deformation in six directions and leads to stress anisotropy.

Figure 1 shows the images of granite debris obtained by a scanning electron microscope with a magnification of $300 x$. The rock was a typical intrusive igneous rock formed by early magmatic activity in Yanshan, and the direction of invasion determines its anisotropy. In rock fracture experiments, stress anisotropy is caused by fracture behaviour.

2.1.2. Heterogeneity. The rock is a collection of minerals with prominent heterogeneity [21]. The granite shown in Figure 1 was porphyritic, where the porphyry crystals were mainly potassium feldspar and quartz. The main minerals were potassium feldspar, plagioclase, and quartz as well as small amounts of biotite and amphibole. This type of granite is called weakly altered medium-to-coarse-grained porphyritic granite. Its mineral composition and corresponding contents are shown in Table 1 . As a magmatic rock, granite is crystalline. It is deformed by a change in lattice bond length and its fracture is the rupture of this bond.

2.1.3. Viscosity. The rock is a typical viscous material [22]. The propagation of $\mathrm{AE}$ signals in it involves the mutual conversion of kinetic and potential energies due to the vibration of rock particles. For AE propagation, the rock prevents the reciprocal motion of particles which leads to a loss of AE energy. For lower energy waveforms, when the energy loss is too large, the component is easily lost. In particular, the high-frequency components of some $\mathrm{AE}$ signals have less energy and are prone to being lost during propagation.

2.2. Analysis of Influential Factors and Model Building. Under the action of deviating stress, the rock can be damaged and can even rupture. AE waveform signals generated by the fracture source are mixed with some reflected, refracted, and overlapping AE signals during propagation, which distorts them. Therefore, signals received by the sensors are divided into four types: (1) source signals of the fracture behaviour of rock, (2) signals distorted by anisotropy, (3) signals distorted by heterogeneity, and (4) signals distorted by noise jamming. According to the physical meaning of wave propagation and the influence of rock properties on wave propagation, equation (1) can be obtained and expressed as follows:

$$
S(t)=\left(1-\xi_{4}(t)\right) *\left(s_{1}(t)+s_{2}(t)+s_{3}(t)+s_{5}(t)\right),
$$

where $S(t)$ represents the AE signals received by a sensor at time $t, s_{1}(t)$ is AE source signal caused by rock fracture at time $t, s_{2}(t)$ is the signal distorted by anisotropy at time $t$, $s_{3}(t)$ is the signal distorted by heterogeneity at time $t, \xi_{4}(t)$ is the signal attenuation coefficient caused by viscosity at time $t$, and $s_{5}(t)$ is noise jamming at time $t$. It is worth noting that $\xi_{4}(t)$ is a quantitative index that describes the signal attenuation during the signal spread through rock media and reflects the viscosity of rock media. Therefore, it can be determined by monitoring the attenuated signal during wave propagation tests.

The temporal sequence of AE reflects the law of energy dissipation during rock fracture and is determined by the size of the fracture as well as the energy stored and released by the rock. The AE frequency domain provides information 


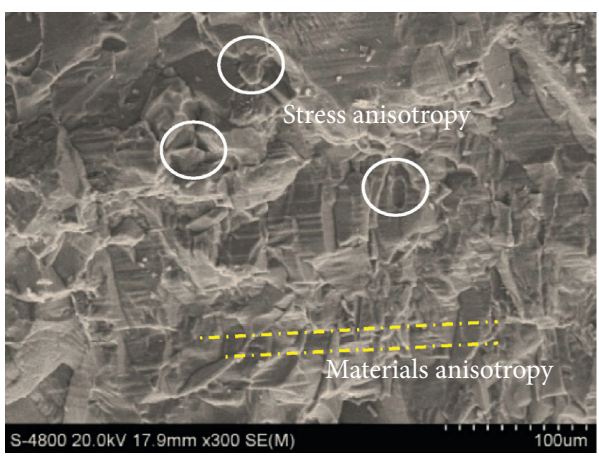

(a)

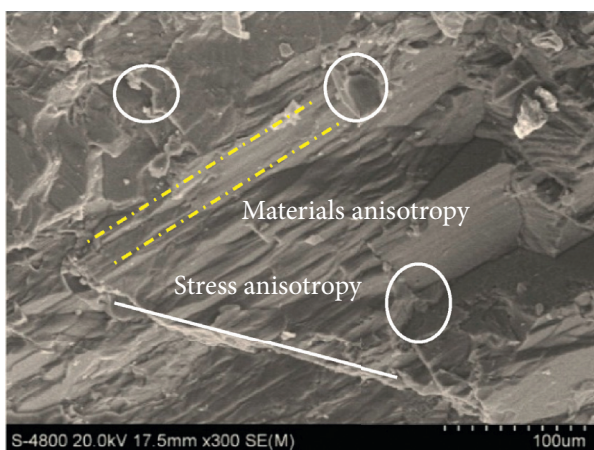

(b)

Figure 1: Images of granite debris obtained by a scanning electron microscope $(\times 300)$. (a) Debris \#1. (b) Debris \#2.

TABLE 1: Composition and corresponding contents of the granite.

\begin{tabular}{|c|c|c|c|c|}
\hline Composition & $\begin{array}{l}\text { Content } \\
(\%)\end{array}$ & Features and structure & $\begin{array}{l}\text { Particle size } \\
\quad(\mathrm{mm})\end{array}$ & Remarks \\
\hline $\begin{array}{l}\text { Potash } \\
\text { feldspar }\end{array}$ & $35 \sim 40$ & Granular & $2-8$ & $\begin{array}{l}\text { Slight kaolinization and sericitization; most of them are } \\
\text { altered along the twin crystal lattices. }\end{array}$ \\
\hline Plagioclase & $25 \sim 30$ & $\begin{array}{l}\text { Visible double crystal and ring } \\
\text { band structure }\end{array}$ & $0.5-3$ & $\begin{array}{c}\text { Different degrees of kaolinization and sericitization; a few } \\
\text { sericites are uncovered. }\end{array}$ \\
\hline Quartz & $30 \sim 35$ & Semiautomorphic to granular & $1.5-8$ & - \\
\hline Biotite & $3 \sim 5$ & Flake & - & - \\
\hline Amphibole & $3 \sim 5$ & Long columnar & - & - \\
\hline
\end{tabular}

about the source of fracture, such as the size of the fracture, the energy dissipation, and the degrees of opening and closing of the fracture surface, especially for the classification and identification of crack patterns. It is one of the most accurate methods to represent the fracture source.
2.2.1. Time Sequence. Signal energy is the area of the signal envelope; that is, the AE signal is integrated there. The time sequence of $\mathrm{AE}$ is expressed from the perspective of energy as follows:

$$
\int_{t-1}^{t} S(t) \mathrm{d} t=\left(1-f_{\xi_{4}}(t, l)\right) *\left(\int_{t-1}^{t} s_{1}(t) \mathrm{d} t-\int_{t-1}^{t} s_{2}(t) \mathrm{d} t+\int_{t-1}^{t} s_{3}(t) \mathrm{d} t+\int_{t-1}^{t} s_{5}(t) \mathrm{d} t\right)+\int_{t-1}^{t} R(n) \mathrm{d} t .
$$

Then,

$$
\left.\begin{array}{l}
E\left(S_{t}\right)=\left(1-\xi_{4}(t)\right) *\left(E\left(s_{1}(t)\right)-E\left(s_{2}(t)\right)-E\left(s_{3}(t)\right)+E\left(s_{5}(t)\right)\right)(1) \\
E\left(S_{t}\right)=\left(1-\xi_{4}(t)\right) *\left(E\left(s_{1}(t)\right)-E\left(s_{2}(t)\right)+E\left(s_{3}(t)\right)+E\left(s_{5}(t)\right)\right)(2)
\end{array}\right\},
$$

where $E\left(S_{t}\right)$ is signal energy received by the sensor at time $t$ and $E\left(s_{1}(t)\right)$ is energy generated by the rupture at time $t$. $s_{1}(t)$ is the main component of the signals and is a positive number $(+) . E\left(s_{2}(t)\right)$ is the reduction in energy due to heterogeneity at time $t$ and is a negative number $(-)$; $E\left(s_{3}(t)\right)$ is the reflection or refraction caused by anisotropy at time $t$. (1) When passing through an open fissure with a degree of opening $D \geq$ wavelength $(\lambda)$, the energy decreases $(-)$. (2) When passing through a closed fissure or an open fissure with degree $D<$ wavelength $(\lambda)$, the energy is superimposed $(+)$. If the filling in the open crack is liquid, longitudinal $\mathrm{AE}$ waves cannot pass through and only transverse waves can. $\xi_{4}(t)$ represents signal attenuation due to viscosity at time $t$ and is positively correlated with the 
propagation distance at time $t,<1 . E\left(s_{5}(t)\right)$ is energy carried by noise at time $t$ and is a positive number $(+)$.
2.2.2. Frequency Domain. We apply the Fourier transform to signal $S(t)$ to determine its frequency distribution:

$$
\begin{aligned}
F(w)= & \int_{-\infty}^{\infty} S(t) e^{-i w t} \mathrm{~d} t=\int_{-\infty}^{\infty} s_{1}(t) e^{-i w t} \mathrm{~d} t+\int_{-\infty}^{\infty} s_{2}(t) e^{-i w t} \mathrm{~d} t+\int_{-\infty}^{\infty} s_{3}(t) e^{-i w t} \mathrm{~d} t+\int_{-\infty}^{\infty} s_{5}(t) e^{-i w t} \mathrm{~d} t=F_{s 1}(w)+F_{s 2}(w) \\
& +F_{s 3}(w)+F_{s 5}(w)
\end{aligned}
$$

where $F(w)$ represents the frequency distribution of $\mathrm{AE}$ signals received by the sensor at time $t, F_{s 1}(w)$ represents the frequency distribution corresponding to rock fracture at time $t, F_{s 2}(w)$ represents signal distortion caused by rock heterogeneity at time $t$ and the change of frequency due to reflection or refraction between crystals, $F_{s 3}(w)$ represents the change in frequency caused by signal distortion due to anisotropy at time $t$, and $F_{s 5}(w)$ represents the frequency corresponding to noise signals at time $t$ and is a superposition effect.

(1) Rock Fracture Behaviour. Rocks of different scales are damaged or destroyed, and the frequency band of AE signals has a positive correlation with the scale of fracture $[23,24]$. For large-scale damage at an engineering site, the frequency is generally distributed in lower band. Rock fracture in the laboratory features small-scale cracks, and the frequency is generally distributed in the higher band. The relationship between the frequency of $\mathrm{AE}$ signals and the crack-related information they convey is as follows:

$$
f \sim \frac{1}{[T+(c / v)(1-(v / \beta) \cos \theta]},
$$

where $T$ is the time of crack closure, opening, or slippage, $c$ is crack length, $v$ is the speed at which a crack is formed, $\theta$ is the angle between the direction of signal propagation and the crack surface, $\theta \in[-\pi, \pi]$, and $\beta$ is the rate of propagation of AE signals in a rock.

$\theta=-\pi$ or $\pi$ is discussed below, and equation (5) can be changed to

$$
f \sim \frac{1}{[T+(c / v)(1-(v / \beta)]}, \quad \theta=-\pi \text { or } f \sim \frac{1}{[T+(c / v)(1+(v / \beta)]}, \quad \theta=\pi .
$$

Equation (6) can be changed as follows:

$$
\frac{1}{f} \sim\left[T + ( \frac { c } { v } ) ( 1 - ( \frac { v } { \beta } ) ] , \quad \theta = - \pi \text { or } \frac { 1 } { f } \sim \left[T+\left(\frac{c}{v}\right)\left(1+\left(\frac{v}{\beta}\right)\right], \quad \theta=\pi\right.\right.
$$

By inserting $c=v \cdot T$ into the above, we get

$$
\frac{1}{f} \sim\left[T+T\left(1-\left(\frac{v}{\beta}\right)\right]\right], \quad \theta=-\pi \text { or } \frac{1}{f} \sim\left[T+T\left(1+\left(\frac{v}{\beta}\right)\right], \quad \theta=\pi\right.
$$

We then make the following transformation:

$\frac{1}{f} \sim T\left(2-\frac{v}{\beta}\right), \quad \theta=-\pi$ or $\frac{1}{f} \sim T\left(2+\frac{v}{\beta}\right), \quad \theta=-\pi$.

$$
\begin{aligned}
& f \sim \frac{1}{(2 T-c / \beta)}, \\
& \theta=-\pi \text { or } f \sim \frac{1}{(2 T+c / \beta)},
\end{aligned}
$$

That is,

$$
\theta=\pi
$$


According to equation (10), the frequency of AE signals generated by rock damage or fracture is related to $T$ (the time it takes for a crack to close, open, or slip) and $c$ (crack length). If a rock material is determined, then $\beta$ is the rate of propagation of $\mathrm{AE}$ signals in a rock and can be regarded as a constant.

We can further transform equation (10), where $v=c / T$ :

$$
f \sim \frac{1}{c} \cdot \frac{1}{(2 / v-1 / \beta)}, \quad \theta=-\pi \text { or } f \sim \frac{1}{c} \cdot \frac{1}{(2 / v+1 / \beta)}, \quad \theta=\pi .
$$

According to equation (11), the frequency distribution of $\mathrm{AE}$ signals generated by rock damage or fracture is inversely proportional to $c$ (the length of the crack); that is, the longer a crack is, the lower the frequency is. The frequency distribution of the AE signals is proportional to $v$ (speed of crack propagation); that is, the faster crack propagation is, the lower the frequency is.

(2) Signal Distortion Caused by Heterogeneity. The rock material is a collection of minerals. As shown in Figure 2, suppose that there are three minerals in a rock: mineral 1 , mineral 2, and mineral 3. The AE source/fracture source generates $\mathrm{AE}$ signals at point $\mathrm{A}$, which propagate to sensors No. 1 and No. 2. Suppose that fracture point A occurs, and the $\mathrm{AE}$ signals received by sensor No. 1 are $F 1=S_{F} 1+S 2+S^{\prime \prime} 3$, that is, three superimposed composite signals. $S_{F} 1$ is the reflected signal when signal $S 1$ meets mineral $3, S 2$ is the signal obtained directly from the fracture source $\mathrm{A}$, and $S^{\prime \prime} 3$ is the signal of $S 3$ that has undergone two interfacial refractions through mineral 2. The AE signals received by sensor No. 2 are obtained by $S 1$, which undergoes two interfacial refractions through mineral 3. Therefore, under the influence of heterogeneity, both $S 1$ and $S 2$ are distorted, and heterogeneity thus has a prominent influence on $\mathrm{AE}$ propagation.

(3) Signal Distortion Caused by Anisotropy. According to reasons for its occurrence, anisotropy can be divided into material anisotropy (rock body) and stress anisotropy (fracture). Material anisotropy is the physical property of the rock medium itself and so is not discussed here.

The existence of deviator stress leads to damage to or the destruction of the rock, which causes stress anisotropy. This leads to damage and fracture in various directions and with differences. Open cracks and sliding, staggered closed cracks are formed, which distort the AE signals. As shown in Figure 3, under the influence of anisotropy, if fracture occurs at point $\mathrm{A}$, the $\mathrm{AE}$ signal received by sensor No. 1 is $F 1=S_{F}^{\prime} 1+S^{\prime} 2$, which is a composite of two superimposed signals. $S_{F}^{\prime} 1$ is the refraction of signal $S_{F} 1$ in an open crack that is obtained when $S 1$ is reflected in a shear-slip crack.

$S^{\prime} 2$ is the refracted signal of $S 2$ in an open crack. The AE signal received by sensor No. 2 is $F 2=S^{\prime} 1+S_{F}^{\prime} 2$, where $S^{\prime} 1$ is the refraction of $S 1$ through a shear-slip crack, $S_{F}^{\prime} 2$ is $S 2$ that first undergoes interfacial reflection from an open crack and then is refracted through a shear-slip crack. In addition, when $S 2$ and $S_{F} 1$ pass through an open crack, they lose

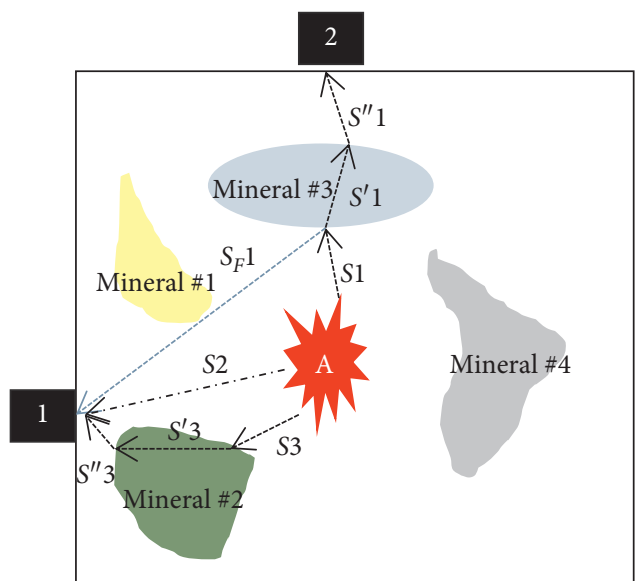

FIGURE 2: Schematic diagram of the influence of heterogeneity on AE signal propagation.

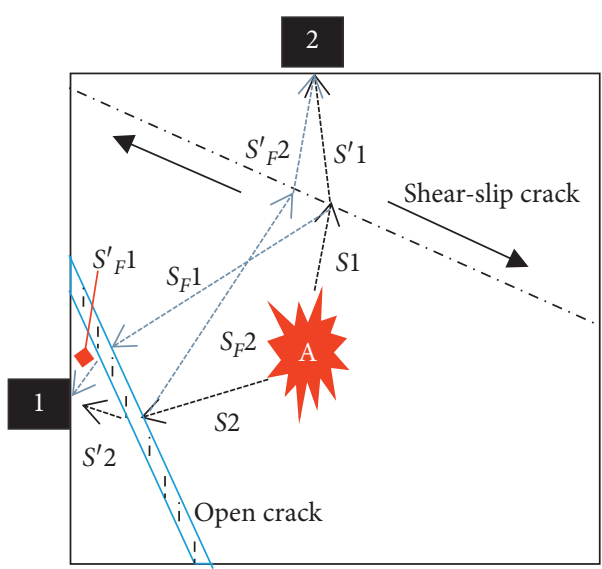

FIgURE 3: Schematic diagram of the influence of anisotropy on AE signal propagation.

energy, which leads to the loss of some high-frequency signals. Under the influence of the anisotropy of rock, the interfacial reflection and refraction of the fracture surface cause signal distortion and energy attenuation. Anisotropy thus has an impact on AE propagation.

(4) Noise Jamming. Noise jamming causes the superimposition of AE signals, which in turn increases the energy and complicates the frequency components. By analysing the noise signals, this interference can be eliminated. In addition, the energy stored in rocks is dissipated, caused by fracture, and some of it is used to overcome the damping force [25]. The viscosity of the rock reduces the energy carried by AE signals.

Assuming that $\mathrm{AE}$ signals travel a unit wavelength, the energy decreases by $\xi$. The factors influencing changes in the AE signals during rock fracture are summarised in Table 2: rock fracture behaviour and signal distortion caused by anisotropy, heterogeneity, viscosity, and noise jamming. 
TABLE 2: Influential factors for and properties of AE signals during rock fracture.

\begin{tabular}{|c|c|c|c|c|}
\hline Impact angle & Factor & Composition of influencing factor & $\begin{array}{l}\text { Symbolic } \\
\text { representation }\end{array}$ & Impact property \\
\hline \multirow{5}{*}{$\begin{array}{l}\text { Frequency } \\
\text { domain }\end{array}$} & $\begin{array}{l}\text { Fracture } \\
\text { behaviour }\end{array}$ & $\begin{array}{l}T \text { (the time it takes for a crack to close, open, or slip) and } c \\
\text { (the length of the crack). If the rock is determined, } \beta \text { is a } \\
\text { fixed value }\end{array}$ & $S_{1}(t)$ & $\begin{array}{c}\text { Composition of a signal } \\
\text { itself }\end{array}$ \\
\hline & Heterogeneity & $\begin{array}{l}\text { When AE signals pass through the boundary of mineral } \\
\text { grains, intergranular reflection or refraction occurs }\end{array}$ & $S_{2}(t)$ & Signal distortion \\
\hline & Anisotropy & $\begin{array}{l}\text { There are open cracks and sliding, staggered closed cracks } \\
\text { that cause the reflection or refraction of AE signals }\end{array}$ & $S_{3}(t)$ & Signal distortion \\
\hline & Viscosity & Rock material itself & $S_{4}(t)$ & Signal distortion \\
\hline & Noise jamming & Signal superposition & $S_{5}(t)$ & Complicated \\
\hline \multirow{5}{*}{$\begin{array}{l}\text { Time } \\
\text { sequence }\end{array}$} & $\begin{array}{c}\text { Rupture } \\
\text { behaviour }\end{array}$ & The energy dissipated during a rupture & $+E\left(S_{1}(t)\right)$ & Energy body \\
\hline & Heterogeneity & The energy carried by reflected or refracted signals & $+E\left(S_{2}(t)\right)$ & Energy superposition \\
\hline & Anisotropy & $\begin{array}{c}\text { The energy carried by reflected or refracted signals. The } \\
\text { energy decreases when the signal passes through an open } \\
\text { crack }\end{array}$ & $\pm E\left(S_{3}(t)\right)$ & $\begin{array}{l}\text { Energy superposition } \\
\text { energy attenuation }\end{array}$ \\
\hline & Viscosity & Rock material itself & $-E\left(S_{4}(t)\right)$ & Energy attenuation \\
\hline & Noise jamming & Environmental factors & $+E\left(S_{5}(t)\right)$ & Energy superposition \\
\hline
\end{tabular}

2.3. Analysis of Stages of Rock Fracture Behaviour. The above models are used to explain the physical mechanism of changes in AE during rock damage and fracture.

(1) In the initial stage (stage 1), only the closure of an original crack occurs inside the rock, and new cracks do not emerge. AE signals are generated by the closure of the original crack, and AE energy in terms of time sequence is determined by its behaviour $E\left(s_{1}(t)\right)$, heterogeneity $E\left(s_{2}(t)\right)$, viscosity $\xi_{4}(t)$, and noise jamming $E\left(s_{5}(t)\right)$. In the frequency domain, it is composed of crack behaviour $s_{1}(t)$, heterogeneity $s_{2}(t)$, and noise jamming $s_{5}(t)$.

$$
\left.\begin{array}{l}
E\left(S_{t}\right)=\left(1-\xi_{4}(t)\right) *\left(E\left(s_{1}(t)\right)+E\left(s_{2}(t)\right)+E\left(s_{5}(t)\right)\right) \text { time sequence } \\
F(w)=F_{s 1}(w)+F_{s 2}(w)+F_{s 5}(w) \quad \text { frequency domain }
\end{array}\right\} .
$$

(2) The elastic stage: AE signals are caused by crystal deformation. AE energy in the time sequence is composed of heterogeneity $E\left(s_{2}(t)\right)$, viscosity $\xi_{4}(t)$, and noise jamming $E\left(s_{5}(t)\right)$. In the frequency domain, it is composed of heterogeneity $s_{2}(t)$ and noise jamming $s_{5}(t)$.

$\left.\begin{array}{l}E\left(S_{t}\right)=\left(1-\xi_{4}(t)\right) *\left(E\left(s_{2}(t)\right)+E\left(s_{5}(t)\right)\right) \quad \text { time sequence } \\ F(w)=F_{s 2}(w)+F_{s 5}(w) \quad \text { frequency domain }\end{array}\right\}$,
(3) The plastic stage: new cracks are formed in the rock. As the loading increases, the cracks penetrate one another to form a macroscopic shear-slip surface or open cracks. AE signals are jointly determined by the fracture behaviour, heterogeneity, anisotropy, viscosity, and noise jamming. It consists of rupture behaviour $E\left(s_{1}(t)\right)$, heterogeneity $E\left(s_{2}(t)\right)$, anisotropy $E\left(s_{3}(t)\right)$, viscosity $\xi_{4}(t)$, and noise jamming $E\left(s_{5}(t)\right)$ in terms of time sequence and is composed of rupture behaviour $s_{1}(t)$, heterogeneity $s_{2}(t)$, anisotropy $s_{3}(t)$, viscosity $\xi_{4}(t)$, and noise jamming $s_{5}(t)$ in terms of the frequency domain.

$$
\left.\begin{array}{l}
E\left(S_{t}\right)=\left(1-\xi_{4}(t)\right) *\left(E\left(s_{1}(t)\right)+E\left(s_{2}(t)\right) \pm E\left(s_{3}(t)\right)+E\left(s_{5}(t)\right)\right) \quad \text { time sequence } \\
F(w)=F_{s 1}(w)+F_{s 2}(w)+F_{s 3}(w)+F_{s 5}(w) \quad \text { frequency domain }
\end{array}\right\} .
$$

(4) Postpeak stage: the rock has failed due to instability and has lost its bearing capacity. The composition of AE signals at this time is similar to that in the plastic phase and is also determined by fracture behaviour, heterogeneity, anisotropy, viscosity, and noise jamming. It is expressed as in the following equation: 


$$
\left.\begin{array}{l}
E\left(S_{t}\right)=\left(1-\xi_{4}(t)\right) *\left(E\left(s_{1}(t)\right)+E\left(s_{2}(t)\right) \pm E\left(s_{3}(t)\right)+E\left(s_{5}(t)\right)\right) \quad \text { time sequence } \\
F(w)=F_{s 1}(w)+F_{s 2}(w)+F_{s 3}(w)+F_{s 5}(w) \quad \text { frequency domain }
\end{array}\right\} .
$$

In short, changes in AE during rock damage and fracture evolution are complex, and factors influencing it in different stages are not the same.

\section{Propagation Characteristics of $\mathbf{A E}$ Waveform Signals}

3.1. Features of AE Waveform Signals. Under the action of external force, rocks with microscopic particles as a unit undergo mechanical movement and accumulate a certain amount of potential energy owing to elastic strain. The occurrence of deviatoric stress causes local deformation or even fracture, and the accumulated elastic strain-based energy is released in the form of elastic waves. The propagation of elastic waves is a process in which energy propagates from the fracture source to the surrounding media, that is, the propagation of AE signals. It can be described according to the theory of elastic waves.

A point in an elastic body and a section with an arbitrary normal direction can be decomposed into normal stress and shear stress in general. When the AE waveform passes through any position in the rock medium, the force affects the process of energy transfer. This effect can be divided into a spherical stress (normal stress) tensor and a deviatoric stress (shear stress) tensor. The spherical stress (normal stress) tensor appears as volumetric deformation and expansion and contraction along the direction of propagation. The form of the movement is a longitudinal wave ( $\mathrm{P}$-wave, expansion and contraction wave). The deviatoric stress (shear stress) tensor appears as shear deformation with a direction of propagation vertical to the direction of motion. It is a transverse wave (S-wave, shear wave). According to the orthogonal plane rule, it can be further divided into an SVwave and an SH-wave (Figure 4).

According to differences in the modes of propagation of different AE wave signals, they can be divided into S-waves and P-waves (Figure 4). The direction of propagation of the $\mathrm{S}$-wave is perpendicular to that of resonance and can be further divided into an SV-wave and an SH-wave that have a deviatoric stress effect and reduce energy. The direction of propagation of the $\mathrm{P}$-wave is consistent with that of resonance, and it forms a spherical stress tensor to reduce energy.

\subsection{Propagation Characteristics of AE Signals in Rocks}

3.2.1. AE Longitudinal and Transverse Waves. In the same medium, the propagation of vertical and horizontal waves is determined by the wave equation, and the process of propagation of $\mathrm{AE}$ signals is shown in the following equation:

$$
\left.\begin{array}{c}
\nabla^{2} \varphi-\frac{1}{v_{P}^{2}} \frac{\partial^{2} \varphi}{\partial t^{2}}=0 \\
\nabla^{2} \psi-\frac{1}{v_{S}^{2}} \frac{\partial^{2} \psi}{\partial t^{2}}=0
\end{array}\right\}
$$

The energy transfer mode of the transverse wave (Swave, shear wave) is shear transfer, and its shape changes into that of a deviatoric stress tensor. The mode of propagation of rock materials is viscous and leads to a loss of energy during the propagation of the transverse wave. A longitudinal wave ( $\mathrm{P}$-wave, expansion and contraction waves) is compressed and expanded along the direction of propagation, which leads to a deformation of volume and has the effect of a spherical stress tensor. For this type of propagation, rock materials exhibit elastic characteristics; that is, the propagation of the longitudinal wave does not lead to the loss of energy.

\subsubsection{Reflection Characteristics of the Free Interface on a Rock Boundary}

(1) P-Wave. As shown in Figure 5, there is no other medium on the surface of the rock boundary, and the P-wave signal $\varphi_{1}(P)$ reflects only along the boundary between air or vacuum and the rock. When the P-wave encounters a rock boundary, it produces positive stress on the rock as well as tangential stress at a certain angle of incidence, which results in waveform conversion to form a reflected wave $\varphi_{2}(P)$ and a reflected wave $\psi_{3}(S V)$.

According to the law of propagation of the P-wave and $\mathrm{S}$-wave in elastic media, we assume that the density of the medium is $\rho$, the longitudinal wave velocity is $v_{P}$, the transverse wave velocity is $v_{S}$, the incident angle is $\alpha$, the 


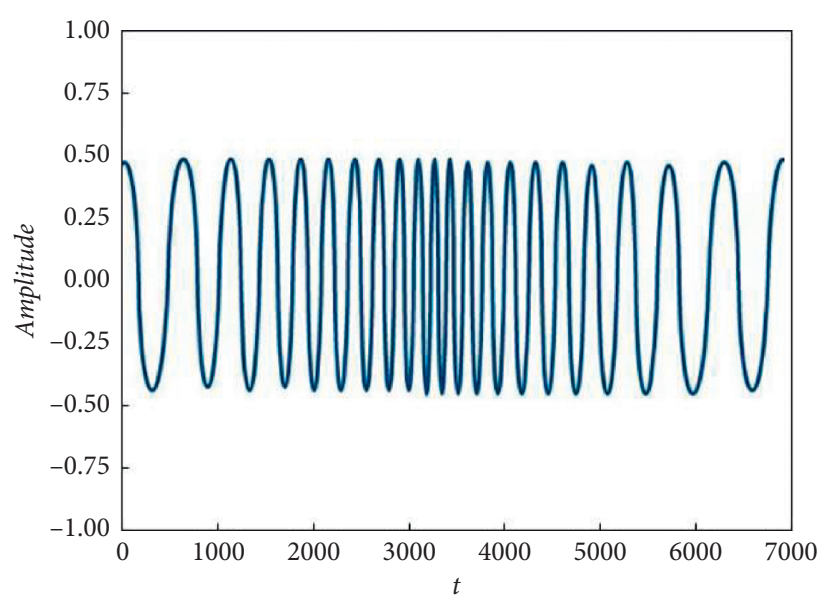

(a)

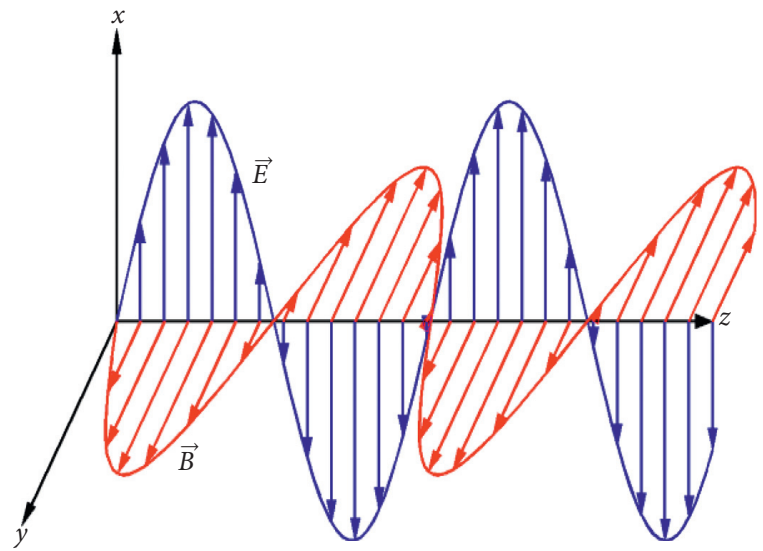

(b)

Figure 4: Classification of AE signal waveform. (a) Longitudinal wave (P-wave). (b) Transverse wave (S-wave).

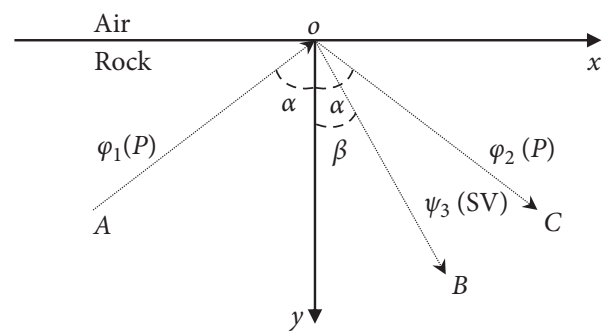

FiguRE 5: Propagation of acoustically emitted P-wave on rock boundaries.

reflection angle of the $\mathrm{P}$-wave is also $\alpha$, and that of the SVwave is $\beta$. The potential functions of the P-wave, reflected $\mathrm{P}$-wave, and reflected SV-wave are then as follows:

$$
\left.\begin{array}{lc}
\varphi_{1}=A_{1} \exp \left[j \omega\left(t-\frac{x \sin \alpha-z \cos \alpha}{v_{P}}\right)\right] & \text { incident } P \text { - wave } \\
\varphi_{2}=A_{2} \exp \left[j \omega\left(t-\frac{x \sin \alpha+z \cos \alpha}{v_{P}}\right)\right] & \text { reflected } P \text { - wave } \\
\psi_{3}=A_{3} \exp \left[j w\left(t-\frac{x \sin \beta+z \cos \beta}{v_{S}}\right)\right] & \text { reflected } S V-\text { wave }
\end{array}\right\} .
$$

The rock is a viscous medium. According to the propagation of the P-wave, the rock exhibits elastic deformation without energy loss. Due to the mode of propagation, the reflected SV-wave has the effect of deviatoric stress accompanied by the loss of energy. Suppose that the incident $\mathrm{P}$-wave is reflected at point $\mathrm{O}\left(x_{O}, y_{O}\right)$ on the rock boundary, forms a reflected SV-wave, and propagates to point $\mathrm{B}\left(x_{B}, y_{B}\right)$. The length of the journey is 
$l_{\mathrm{OB}}=\sqrt{\left(x_{B}-x_{O}\right)^{2}+\left(y_{B}-y_{O}\right)^{2}}$ and the velocity of the SVwave is $v_{S V}$; then, the loss of energy is $\Pi_{\mathrm{SV}}=l_{\mathrm{OB}} / v_{\mathrm{SV}} \xi$ and $\xi$ is termed the damage factor. Moreover, $\xi$ is a time-varying factor that describes the energy loss during signal propagation because of fracturing evolution.

The relationship between the energies carried by the incident $\mathrm{P}$-wave, reflected $\mathrm{P}$-wave, reflected $\mathrm{SV}$-wave, and the attenuation of the SV-wave is as follows:

$$
\mathrm{E}_{1}=E_{2}+E_{3}+\Pi_{\mathrm{SV}}
$$

(2) $S$-Wave. As shown in Figure 6, the SH-wave $\psi_{1}(\mathrm{SH})$ is reflected only on the boundary. According to the propagation mode of the $S$ wave, mode conversion does not occur, and only the reflected $\mathrm{SH}$-wave $\psi_{2}(\mathrm{SH})$ exists.

Due to the law of propagation of the S-wave, suppose that the medium density is $\rho$, velocity of the transverse wave is $v_{S}$, and the incident angle and the reflection angle are both $\beta$. The potential functions of the incident and reflected $\mathrm{SH}-$ wave are then as follows:

$$
\left.\begin{array}{l}
\psi_{1}=B_{1} \exp \left[j w\left(t-\frac{x \sin \beta-z \cos \beta}{v_{S}}\right)\right] \quad \text { incident SH - waves } \\
\psi_{2}=B_{2} \exp \left[j w\left(t-\frac{x \sin \beta+z \cos \beta}{v_{S}}\right)\right] \quad \text { reflected SH - wave }
\end{array}\right\} .
$$

Assuming that the incident $\mathrm{SH}$-wave propagates from the fracture source $\mathrm{A}\left(x_{A}, y_{A}\right)$ to the rock boundary $O\left(x_{O}, y_{O}\right)$, the length of travel is $l_{\mathrm{AO}}=\sqrt{\left(x_{A}-x_{O}\right)^{2}+\left(y_{A}-y_{O}\right)^{2}}$. The reflected SH-wave propagates from point $O$ to point $B\left(x_{B}, y_{B}\right)$, where the distance travelled is $l_{O B}=\sqrt{\left(x_{0}-x_{B}\right)^{2}+\left(y_{0}-y_{B}\right)^{2}}$, and the velocity the of incident $\mathrm{SH}$-wave and the reflected $\mathrm{SH}$-wave is $v_{\mathrm{SH}}$. Then, the loss of energy is $\Pi_{\mathrm{SH}}=\Pi_{\text {incident SH wave }}{ }^{+}$ $\Pi_{\text {reflected SH wave }}=l_{A O} / v_{\mathrm{SH}}+l_{\mathrm{OB}} / v_{\mathrm{SH}}=l_{A O}+l_{\mathrm{OB}} / \nu_{\mathrm{SV}}$.

The incident $\mathrm{SH}$-wave propagates from point $A$ to point $O$ on the rock boundary, and the reflected $\mathrm{SH}$-wave forms and propagates from point $O$ to point $B$. The relationship between the energies carried by the incident $\mathrm{SH}$-wave and the reflected $\mathrm{SH}$-wave and the attenuation in the $\mathrm{SH}$-wave is as follows:

$$
E_{1}=E_{2}+E_{3}+\Pi_{\mathrm{SH}} \cdot
$$

(3) Propagation Characteristics of the Wave along the RockGrain Boundary. The important physical properties of rock minerals are their density, Poisson's ratio, and Young's modulus. Different mineral particles form rock materials through cementation or crystallization, and the rate of propagation of AE signals in different minerals is different. When it encounters mineral boundaries, the waveform is reflected or refracted. We discuss this separately for P-waves and S-waves.

P-wave: the signal $\varphi_{1}(P)$ of the $\mathrm{P}$-wave is subjected to reflection, refraction, and waveform conversion, resulting in the reflected $\mathrm{P}$-wave $\varphi_{2}(P)$, reflected SV-wave $\psi_{3}(\mathrm{SV})$, refracted $\mathrm{P}$-wave $\varphi_{4}(P)$, and refracted $\mathrm{SV}$-wave $\psi_{4}(\mathrm{SV})$ (Figure 7).

We assume that the density of mineral $\mathrm{I}$ is $\rho_{1}$, its Poisson's ratio is $\lambda_{1}$, the velocity of the longitudinal wave is $v_{P 1}$, the velocity of the transverse wave is $v_{S 1}$, the density of mineral II is $\rho_{2}$, its Poisson's ratio is $\lambda_{2}$, the velocity of the longitudinal wave is $v_{P 2}$, and the velocity of the transverse wave is $v_{S 2}$. Suppose that the incident angle of the $\mathrm{P}$-wave is $\alpha$, its reflected angle is also $\alpha$, the reflected angle of the SVwave is $\beta$, its refraction angle is $\alpha^{\prime}$, and the refraction angle of the SV-wave is $\beta^{\prime}$. The relationship between the energies carried by the incident $\mathrm{P}$-wave, reflected $\mathrm{P}$-wave, reflected $\mathrm{SV}$-wave, refracted $\mathrm{P}$-wave, and refracted $\mathrm{SV}$-wave is as follows: 


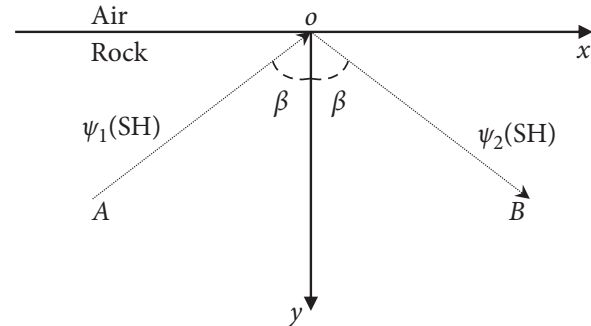

Figure 6: Propagation of acoustically emitted S-wave on rock boundaries.

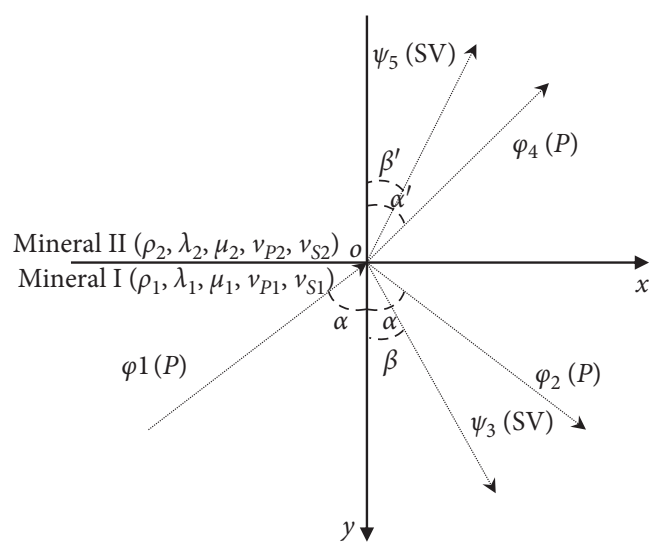

Figure 7: Propagation of AE P-wave on mineral boundaries.

$$
\begin{aligned}
& \varphi_{1}=A_{1} \exp \left[j w\left(t-\frac{x \sin \alpha-z \cos \alpha}{v_{P 1}}\right)\right] \text { incident } P \text { wave } \\
& \varphi_{2}=A_{2} \exp \left[j w\left(t-\frac{x \sin \alpha+z \cos \alpha}{v_{P 1}}\right)\right] \quad \text { reflected } P \text { wave } \\
& \left.\psi_{3}=A_{3} \exp \left[j w\left(t-\frac{x \sin \beta+z \cos \beta}{v_{S 1}}\right)\right] \text { reflected SV wave }\right\} \\
& \varphi_{4}=A_{4} \exp \left[j w\left(t-\frac{x \sin \alpha^{\prime}+z \cos \alpha^{\prime}}{v_{P 2}}\right)\right] \quad \text { refracted } \mathrm{P} \text { wave } \\
& \psi_{4}=A_{5} \exp \left[j w\left(t-\frac{x \sin \beta^{\prime}+z \cos \beta^{\prime}}{v_{S 2}}\right)\right] \quad \text { refracted SV wave }
\end{aligned}
$$

According to rock viscosity, the reflected SV-wave and refracted SV-wave lose energy during propagation. Suppose that the incident $\mathrm{P}$-wave is reflected and refracted at point $O\left(x_{O}, y_{O}\right)$ on the mineral boundary, the reflected SV-wave $\psi_{3}$ propagates to point $A\left(x_{3}, y_{3}\right)$, and the refracted SV-wave $\psi_{4}$ propagates to point $B\left(x_{4}, y_{4}\right)$. The length of the reflected SV-wave is $l_{O A}=\sqrt{\left(x_{0}-x_{A}\right)^{2}+\left(y_{0}-y_{A}\right)^{2}}$ and that of the refracted SV-wave is $l_{O B}=\sqrt{\left(x_{0}-x_{B}\right)^{2}+\left(y_{0}-y_{B}\right)^{2}}$; then, the total loss of energy is $\Pi_{\mathrm{SV}}=\Pi_{\text {reflected SV wave }}+\Pi_{\text {refracted SV wave }}=l_{\mathrm{OA}} / v_{S 1}+l_{\mathrm{OB}} / v_{S 2}$.

When the incident $\mathrm{P}$-wave propagates to the $\mathrm{AE}$ source/ fracture source and then to point $O$ of the mineral boundary, reflected and refracted P-waves form, and the mode of conversion changes to a reflected SV-wave and a refracted SV-wave. The relationship of the total energy is as follows:

$$
E_{1}=E_{2}+E_{3}+E_{4}+E_{5}+\Pi_{\mathrm{SV}} .
$$


S-wave: according to the propagation characteristics of the $\mathrm{S}$-wave, mode conversion does not occur. As shown in Figure 8 , on the mineral boundary point $O$, the $\mathrm{SH}$-wave is $\psi 1(\mathrm{SH})$, reflected wave is $\psi 2(\mathrm{SH})$, and refracted wave is $\psi 3$ (SH).

Assume that the density of mineral I is $\rho_{1}$, its Poisson's ratio is $\lambda_{1}$, the velocity of the longitudinal wave is $v_{P 1}$, and the velocity of the transverse wave is $v_{S 1}$. The density of mineral II is $\rho_{2}$, its Poisson's ratio is $\lambda_{2}$, the velocity of the longitudinal wave is $v_{P 2}$, and the velocity of the transverse wave is $v_{S 2}$. Suppose that the incident angle of the SH-wave $\psi_{1}$ is $\beta$, the reflected angle of $\mathrm{SH}$-wave $\psi_{2}$ is $\beta$, and the refracted angle of $\mathrm{SH}$-wave $\psi_{3}$ is $\beta^{\prime}$. The potential function of each wave component is then as follows:

$$
\begin{aligned}
& \psi_{1}=A_{1} \exp \left[j w\left(t-\frac{x \sin \beta-z \cos \beta}{v_{S 1}}\right)\right] \quad \text { incident S wave } \\
& \psi_{2}=A_{2} \exp \left[j w\left(t-\frac{x \sin \beta+z \cos \beta}{v_{S 1}}\right)\right] \quad \text { reflected SH wave } \\
& \psi_{3}=A_{3} \exp \left[j w\left(t-\frac{x \sin \beta^{\prime}+z \cos \beta^{\prime}}{v_{S 2}}\right)\right] \quad \text { refracted SH wave } \\
& \begin{cases}\lambda \geq D, & \text { signals can pass through a crack, } \\
\lambda<D, & \text { signals cannot pass through a crack. }\end{cases}
\end{aligned}
$$
fracture source/AE source point $A\left(x_{A}, y_{A}\right)$ to the mineral boundary $O\left(x_{O}, y_{O}\right)$, where its length is $l_{A O}=\sqrt{\left(x_{A}-x_{O}\right)^{2}+\left(y_{A}-y_{O}\right)^{2}}$. The reflected SH-wave propagates from point $O$ to point $B\left(x_{B}, y_{B}\right)$ over distance $l_{O B}=\sqrt{\left(x_{0}-x_{B}\right)^{2}+\left(y_{0}-y_{B}\right)^{2}}$, and the refracted SH-wave propagates from point $O$ to point $C\left(x_{C}, y_{C}\right)$ over distance $l_{O C}=\sqrt{\left(x_{0}-x_{C}\right)^{2}+\left(y_{0}-y_{C}\right)^{2}}$. Then, the total loss of energy is $\quad \Pi_{\mathrm{SH}}=\Pi_{\text {Incident SH wave }}$ $+\prod_{\text {Reflected SH wave }}+\Pi_{\text {Refracted SH wave }}=l_{A O} /$ $v_{S 1}+l_{O B} / v_{S 1}+l_{O C} / v_{S 2}$.

The incident $\mathrm{SH}$-wave propagates from point $A$ to point $O$ on the rock boundary, and the reflected $\mathrm{SH}$-wave forms and propagates from point $O$ to point $B$. The refracted $\mathrm{SH}$ wave propagates from point $O$ to point $C$. The relationship between the energies carried by the incident $\mathrm{SH}$-wave, reflected $\mathrm{SH}$-wave, refracted $\mathrm{SH}$-wave, and the attenuation of the $\mathrm{SH}$-wave is as follows:

$$
E_{1}=E_{2}+E_{3}+\Pi_{\mathrm{SH}}
$$

(4) Propagation Characteristics of AE Signals through Cracks. According to such physical phenomena as opening, closing, and filling, cracks can be divided into open cracks, openfilled cracks, and closed cracks. For these three types of cracks, the propagation of the $\mathrm{P}$-wave and $\mathrm{S}$-wave is discussed separately.

\section{(i) Open cracks}

Such cracks are not filled with any material. Assuming that the open surface of the crack is $D$, whether the AE waveform can pass through the crack is determined by its wavelength $\lambda$ : (ii) Open-filled cracks

Such cracks have different effects on the propagation of the P-wave and S-wave according to the difference in the filling materials. If the filling is solid, it can be analysed using equation (3); if the filling is liquid, its shear modulus is zero, and it does not return to its original shape under shear stress; that is, it is not shear-resistant. In general, liquids cannot pass through transverse waves.

(iii) Closed fissure

The AE waveform is reflected and refracted here. In addition, the $\mathrm{P}$-wave undergoes waveform conversion, and reflected and refracted SV-waves occur.

\section{Discussion}

The time-frequency characteristics of $\mathrm{AE}$ are determined here by using the stress or deformation of rocks. According to equation (11), the frequency distribution of AE signals is inversely proportional to $c$ (crack length) and directly proportional to $v$ (crack growth rate). This is why ultra-lowfrequency seismic signals occur in case of ultra-large-scale fractures, such as in earthquakes [26, 27], low-frequency microseismic signals occur in case of earthquakes in mines that lead to rock failure, and higher-frequency signals occur in laboratory experiments [28, 29].

AE technology is an effective method for characterising rock damage or fracture. The material characteristics of rocks, such as heterogeneity, anisotropy, and viscosity, as well as the stages of crack evolution can distort the results of monitoring. The complexity of the rock medium and its fracture also lead to complex AE waveform propagation. In 


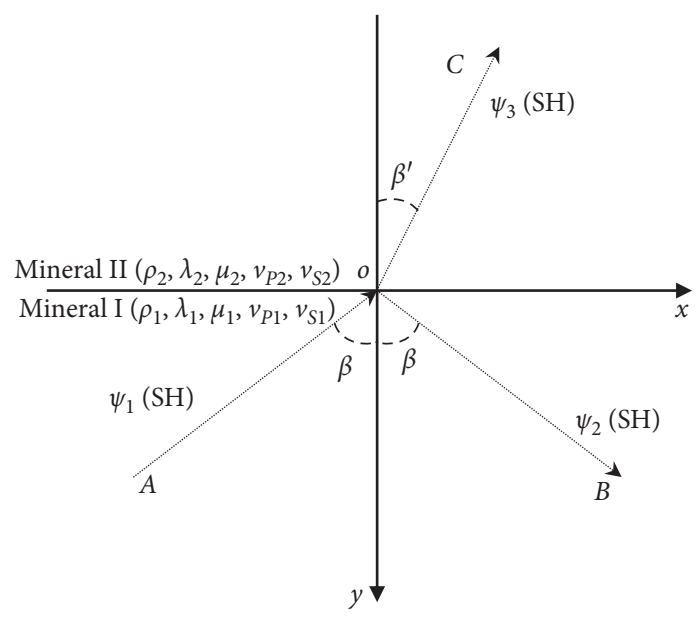

FIGURE 8: Propagation of AE S-wave on mineral boundaries.

addition, different boundary conditions lead to different AE signals.

The characteristics and transmission of $\mathrm{AE}$ during rock damage or fracture caused by multiple factors (damage fracture, loading condition, lithology, size, structure, and moisture) affect the results of analysis. Signals received by a sensor can still reflect the essential characteristics of rock damage and fracture.

\section{Conclusions}

In this study, the effects of the physical properties of rocks on AE waveform signals are examined from the perspective of the rock material and its fracture characteristics as well as the characteristics of the propagation of different $\mathrm{AE}$ waveform signals. The major findings and conclusions can be summarised as follows:

(1) The time-frequency characteristics of AE show the complex rules during rock deformation or fracture and are affected by its heterogeneity, anisotropy, and viscosity as well as noise jamming. Meanwhile, they are also influenced by the fracture morphology and rock structure. The frequency $(f)$ of the AE signals generated by rock fracture is inversely proportional to crack length $(c)$ and directly proportional to the rate of crack growth $(v)$.

(2) The factors influencing the characteristics and propagation of AE signals are varied. Among them, the elastic phase is affected by heterogeneity, viscosity, and noise jamming. During signal propagation, the comprehensive effects of such factors weaken the energy of the signals and enhance the distribution of signal frequency.

(3) A mathematical model has been developed for analysing the propagation of AE signals based on the fracture behaviour of rocks, and their anisotropy, heterogeneity, and viscosity as well as environmental noise jamming by considering the differences in the wave equation of its components. Namely, the AE wave can be divided into a transverse wave (S-wave, shear wave) and a longitudinal wave (P-wave, expansion and contraction wave), and they can be considered, respectively, in the mathematical model.

\section{Data Availability}

The scanned images of granite debris and the composition data of the granite used to support the findings of this study are included within the article.

\section{Conflicts of Interest}

The authors declare no conflicts of interest.

\section{Acknowledgments}

This research was funded by the China Postdoctoral Science Foundation (Grant no. 2020M680950) and the National Natural Science Foundation of China (Grant no. 51627804).

\section{References}

[1] S. Cherny, D. Chirkov, V. Lapin et al., "Two-dimensional modeling of the near-wellbore fracture tortuosity effect," International Journal of Rock Mechanics and Mining Sciences, vol. 46, no. 6, pp. 992-1000, 2009.

[2] J. Wang, Y. Cao, G. Song, and H. Liu, "Diagenetic evolution and formation mechanisms of high-quality reservoirs under multiple diagenetic environmental constraints: an example from the paleogene beach-bar sandstone reservoirs in the dongying depression, bohai bay basin," Acta Geologica SinicaEnglish Edition, vol. 91, no. 1, pp. 232-248, 2017.

[3] S. Hurwitz, K. L. Kipp, S. E. Ingebritsen, and M. E. Reid, "Groundwater flow, heat transport, and water table position within volcanic edifices: implications for volcanic processes in the Cascade Range," Journal of Geophysical Research Solid Earth, vol. 108, no. 12, 2003.

[4] L. Haifeng, Y. Duoxi, L. Xiuyu, G. Liquan, and S. Dan, "Analytical solution of stress in a transversely isotropic floor rock mass under mining," Chinese Journal of Underground Space and Engineering, vol. 9, no. 5, pp. 1050-1056, 2013. 
[5] Z. Chen, "Base features of rock mechanics aspects of underground projects," Journal of China Coal Society, vol. 12, no. 4, pp. 1-7, 1986.

[6] S. G. Lekhnitskii, Theory of Elasticity of an Anisotropic Elastic Body, Holdcnn-Day Inc., San Francisco, CA, USA, 1963.

[7] C. Wengui and Y. Ronggui, "Back analysis of stress measurement of anisotropic rock mass," Journal of China Coal Society, vol. 19, no. 2, pp. 122-130, 1994.

[8] S. Xie and Z. Bao, "Fractal and multifractal properties of geochemical fields," Mathematical Geology, vol. 36, no. 7, pp. 847-864, 2004.

[9] F. Tonon and B. Amadei, "Stresses in anisotropic rock masses: an engineering perspective building on geological knowledge," International Journal of Rock Mechanics and Mining Sciences, vol. 40, no. 7-8, pp. 1099-1120, 2003.

[10] W. C. Zhu, M. S. Zhang, H. X. Zhang, X. Q. Guo, and G. Kai, "Numerical simulation for determining the size of representative element volume (rev) of jointed rock mass," Chinese Journal of Geotechnical Engineering, vol. 35, no. 6, pp. 11211127, 2013.

[11] R. H. Tatham, "Vp/Vs and lithology," Geophysics, vol. 47, no. 3, pp. 336-344, 1982.

[12] D. Y. Xi, Y. P. Liu, X. Y. Liu, Y. M. Shan, and W. G. Liu, "Influence of fatigue loading on physical mechanical characteristics of rock," Chinese Journal of Geotechnical Engineering, vol. 23, no. 3, pp. 292-295, 2001.

[13] D. Y. Xi, B. Liu, and X. Y. Tian, "Anisotropy and nonlinear viscoelastic behavior of saturated rocks," Chinese Journal of Geophysics, vol. 45, no. 1, pp. 109-118, 2002.

[14] T. Deng and L. D. Yang, "Characteristics of velocity ratio of P-wave and S-wave for anisotropic rocks," Chinese Journal of Rock Mechanics and Engineering, vol. 25, no. 10, pp. 20232029, 2006.

[15] W. H. Shi, T. H. Yang, P. T. Wang, G. J. Hu, and P. Xiao, "Anisotropy analysis method for stability of open-pit slope rock mass and its application," Chinese Journal of Geotechnical Engineering, vol. 36, no. 10, pp. 1924-1933, 2014.

[16] X. H. Huang, Y. B. Zhang, B. Z. Tian, and X. X. Liu, "Time delay estimation and acoustic emission source location of rock based on phase difference," Rock and Soil Mechanics, vol. 36, no. 2, pp. 381-386, 2015.

[17] K. Mogi, Study of Elastic Shocks Caused by the Fracture of Better O-Generous Material and its Relation to Earthquake Phenomena, Vol. 40, Bull of the Earthquake Res. Inst., Tokyo, Japan, 1962.

[18] S. H. Wang, Y. B. Zhang, N. Zhang, and C. Lee, "Experimental study on acoustic emission measurement and source location for anisotropic rock failure process," Journal of Northeastern University (Natural Science), vol. 28, no. 7, pp. 1033-1036, 2007.

[19] D. Amitrano, M. Arattano, M. Chiarle et al., "Microseismic activity analysis for the study of the rupture mechanisms in unstable rock masses," Natural Hazards and Earth System Sciences, vol. 10, no. 4, pp. 831-841, 2010.

[20] J. Tang and G. C. Wu, "Stress-dependent anisotropy of mudstone and shale with low porosity," Chinese Journal of Geophysics, vol. 8, pp. 2986-2995, 2015.

[21] M. Menzies, "Alkaline rocks and their inclusions: a window on the Earth's interior," Geological Society, London, Special Publications, vol. 30, no. 1, pp. 15-27, 1987.

[22] S. R. Pride, E. Tromeur, and J. G. Berryman, "Biot slow-wave effects in stratified rock," Geophysics, vol. 67, no. 1, pp. 271-281, 2002.
[23] N. A. Haskell, "Total energy and energy spectral density of elastic wave radiation from propagating faults," Bulletin of the Seismological Society of America, vol. 54, no. 6A, pp. 18111841, 1964.

[24] M. Ohnaka, "A physical basis for earthquakes based on the elastic rebound model," Bulletin of the Seismological Society of America, vol. 66, no. 2, pp. 433-451, 1976.

[25] H. Xie, L. Li, Y. Ju, R. Peng, and Y. Yang, "Energy analysis for damage and catastrophic failure of rocks," Science China Technological Sciences, vol. 54, no. S1, pp. 199-209, 2011.

[26] J. Chan and D. R. Schmitt, "Initial seismic observations from a deep borehole drilled into the Canadian Shield in Northeast Alberta," International Journal of Earth Sciences, vol. 104, no. 6, pp. 1-14, 2014.

[27] G. R. Toro and R. K. Mcguire, "An investigation into earthquake ground motion characteristics in Eastern North America," Bulletin of the Seismological Society of America, vol. 77, no. 2, pp. 468-489, 1987.

[28] C. W. Li, S. Fu, C. Guan, T. Wan, and B. Xie, "Characteristics and generation mechanism of ULF magnetic signals during coal deformation under uniaxial compression," Journal of Geophysics \& Engineering, vol. 15, no. 4, p. 4, 2018.

[29] X. Liu, L. Wu, Y. Zhang, Z. Liang, X. Yao, and P. Liang, "Frequency properties of acoustic emissions from the dry and saturated rock," Environmental Earth Sciences, vol. 78, no. 3, p. 67, 2019. 